\title{
Wave Equation Inversion of Skeletonized Surface Waves
}

Zhen-dong Zhang*, King Abdullah University of Science and Technology, Institute of Geology and Geophysics, Chinese Academy of Sciences, Yike Liu, Institute of Geology and Geophysics, Chinese Academy of Sciences, Gerard Schuster, King Abdullah University of Science and Technology

\section{SUMMARY}

We present a surface-wave inversion method that inverts for the S-wave velocity from the Rayleigh dispersion curve for the fundamental-mode. We call this wave equation inversion of skeletonized surface waves because the dispersion curve for the fundamental-mode Rayleigh wave is inverted using finitedifference solutions to the wave equation. The best match between the predicted and observed dispersion curves provides the optimal S-wave velocity model. Results with synthetic and field data illustrate the benefits and limitations of this method.

\section{INTRODUCTION}

Waveform inversion estimates the velocity model that minimizes the misfit between the predicted and recorded data. However, the data-misfit function can be very sensitive to the accurate prediction of amplitudes, which is difficult to achieve with modeling methods that do not fully take into account viscoelastic and anisotropic effects. Moreover, a poor starting model will promote cycle skipping and convergence to a local minimum (Virieux and Operto, 2009).

To mitigate these problems, other types of data can be inverted that can be more accurately modeled and might enjoy a more quasi-linear relationship between the model and the data. For example, the traveltime misfit function is much less bumpy than the waveform misfit function. We can think of the less complex traveltimes as data skeletonized from the more complicated seismograms. The strategy of Luo and Schuster $(1991 a, b)$ is to invert the skeletonized data to get close to the global minimum with no cycle skipping.

We now adapt the skeletonized inversion strategy to inverting the dispersion curve of the fundamental Rayleigh wave. Instead of picking traveltimes we pick the dispersion curve of the fundamental mode in the frequency-wavenumber domain and invert it for the S-wave velocity model. This procedure is denoted as skeletonized wave equation inversion of surface waves because the elastic wave equation is used to invert the velocity model from the dispersion curves. It can also invert for $2 \mathrm{D}$ or $3 \mathrm{D} \mathrm{S}$-wave velocity models compared to the $1 \mathrm{D}$ layered model in standard surface-wave inversion (Park et al., 1998). It also differs from the waveform inversion approach of Baumstein et al. (2011) and Solano et al. (2014) who invert all of the surface-wave events or windowed portions for the near-surface velocity information. Their approach attempts to explain most of the observed waveforms with predicted ones, and so there still exists the possibility of getting stuck in a local minimum with a poor starting model. This is less of a problem

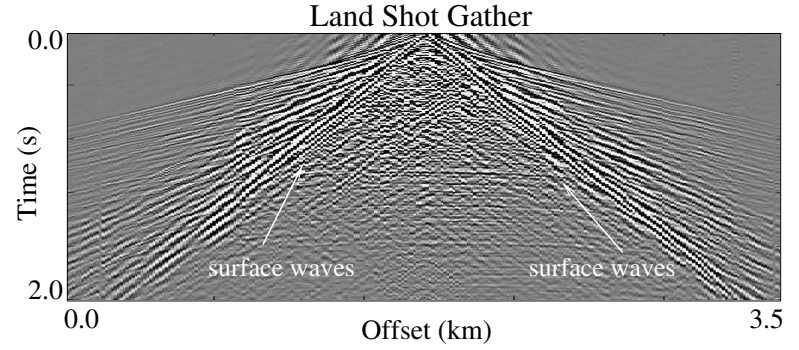

Figure 1: Common shot gather recorded by a land survey.

with skeletonized inversion because it only attempts to explain the simple fundamental dispersion curve. However, if the media is too complex then the fundamental dispersion curve cannot be easily identified and so skeletonized inversion cannot be easily implemented.

This paper is divided into four sections. After the introduction, the theory of skeletonized inversion of surface waves is described in the second section. Numerical results for synthetic and field data are presented in the third section, and the conclusions are in the last section.

\section{THEORY}

We now present the theory for inverting the fundamental dispersion curve for the S-wave velocity model.

In Figure 1, there are many surface-wave cycles in the traces and so the waveform-misfit function for these data are likely to be highly nonlinear with respect to changes in the shear velocities. Therefore we should look for a means to simplify the data. One such reduction is to estimate the dispersion velocity $C(\omega)^{o b s}=\omega / k(\omega)$ curve for the fundamental mode in Figure $2 \mathrm{~b}$, and invert it for the shear-velocity distribution. The locus of points for the fundamental mode is often identified by the maximum amplitudes (Gabriels et al., 1987; Park et al., 1998; Jianghai et al., 1999; Dong et al., 2014) in the spectra with the closest proximity to the $C(\omega)$ (or wavenumber) axis in Figure 2. A surface-wave inversion algorithm that uses the wave equation and an iterative gradient optimization algorithm to invert the dispersion curve is now presented, assuming the fundamental dispersion velocity $C(\omega)^{o b s}$ has been picked from the recorded data.

1. Form the misfit function $\varepsilon$

$$
\varepsilon=1 / 2 \sum_{\omega}\left(C(\omega)-C(\omega)^{o b s}\right)^{2}+1 / 2 R(c(\mathbf{x}))^{2},
$$


where $R(c(\mathbf{x}))$ is the regularization term, $c(\mathbf{x})$ is the $\mathrm{S}$ wave velocity model, and $C(\omega)^{\text {obs }}$ describes the phasevelocity curve for the observed fundamental mode. Here, $C(\omega)$ is the fundamental phase-velocity function predicted by solving the wave equation in the space-time domain and Fourier transforming a shot gather into the $\omega-k$ domain. This predicted curve is determined by manual picking or an automatic method that identifies the maximum amplitudes that are closest to the the $k$ axis to get $k(\omega)^{o b s}$, and using the formula $C(\omega)^{o b s}=$ $\omega / k(\omega)^{o b s}$ to get the phase-velocity curve. In this paper, we use a roughness regularization term $R(c(\mathbf{x}))=$ $\frac{d c(\mathbf{x})}{d z}$ that penalizes sharp jumps in the $\mathrm{S}$-wave velocity model. There are other methods for extracting the dispersion curve, such as the slowness-frequency transform of McMechan and Yedlin (1981) or the F-K method of Park et al. (1998).

2. The steepest descent formula

$$
\begin{aligned}
& c(\mathbf{x})^{(\mathbf{k}+\mathbf{1})}=\mathbf{c}(\mathbf{x})^{(\mathbf{k})}-\alpha \gamma(\mathbf{x}), \\
& =c(\mathbf{x})^{(\mathbf{k})}-\alpha \sum_{\omega} \Delta C(\omega)^{(k)} \frac{\partial C(\omega)}{\partial c(\mathbf{x})}-\beta \frac{\partial R(\mathbf{x})}{\partial c(\mathbf{x})},
\end{aligned}
$$

is used for reconstructing the S-wave velocity profile $c(\mathbf{x})$, where the regularization damping parameter is $\beta$. Here, the data residual is defined as $\Delta C(\omega)^{(k)}=$ $C(\omega)-C(\omega)^{o b s}$. In practice, we recommend a preconditioned conjugate gradient method.

3. For an N-layer medium, there are $N+1$ unknown $\mathrm{S}$ velocity values so that the Fréchet derivative $\frac{\partial C(\omega)}{\partial c(\mathbf{x})}$ can be computed by the finite-difference formula:

$$
\frac{\partial C(\omega)}{\partial c_{i}(\mathbf{x})} \approx \frac{C(\omega)_{c(\mathbf{x})+\delta \mathbf{c}_{\mathbf{i}}(\mathbf{x})}-C(\omega)_{c(\mathbf{x})}}{\delta c_{i}(\mathbf{x})},
$$

where $c(\mathbf{x})$ represents the reference S-wave velocity model and $\delta c_{i}(\mathbf{x})$ is the perturbed velocity in the $i^{t h}$ layer. Two finite-difference simulations are required to compute $\frac{\partial C(\omega)}{\partial c_{i}(\mathbf{x})}$, one for the reference S-wave velocity model and one for the reference model with the $S$-wave velocity in the $i^{t h}$ layer perturbed by $\delta c_{i}(\mathbf{x})$. The shot gathers from these two simulations in the space-time domain are FK transformed to get the two dispersion spectra, the fundamental dispersion curves are identi-

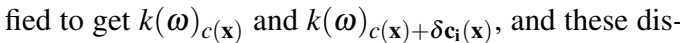
persion curves are used to get the phase velocity curves

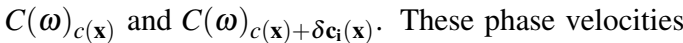
are then inserted into equation 3 to get the approximation to the Fréchet derivative. The normalized residual curve $\Delta C(\omega)^{(k)}$ is also computed and equation 2 is used to update the $\mathrm{S}$ velocity. The fundamental mode is largely insensitive to the P-velocity and density variations (Aki and Richards, 1980), so their values are not iteratively updated. For an $N-1$ layer velocity model, only $N+1$ finite-difference simulations are computed for one shot at each iteration.

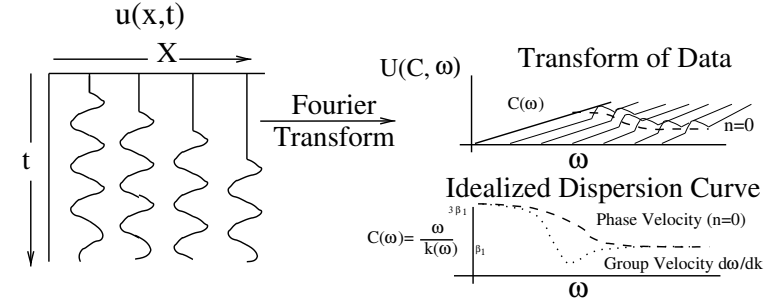

Figure 2: Common shot gather on the left, and right figures depict the (top right) actual $C(\omega)-\omega$ spectrum of the data and (bottom right) idealized dispersion curve for the fundamental Rayleigh mode $(n=0)$ for a two-layered elastic medium with a free surface. Here the dispersion velocity is $C(\omega)=\omega / k(\omega)$.

\section{NUMERICAL EXAMPLE}

\section{Inversion of Synthetic Data}

An example of inverting for the $\mathrm{S}$-wave velocity distribution by skeletonized surface-wave inversion is now presented. A shot gather is computed by a finite-difference solution to the 2D elastic wave equation and is shown in Figure $3 \mathrm{a}$ for the 3-layer model in Figure $3 \mathrm{f}$ (red line). Here, the traces are the vertical-component particle-velocity measurements on the free surface. The source is simulated as an explosive point source in the $2 \mathrm{D}$ modeling code. An FK transform is applied to the shot gather to give the spectrum shown in Figure $3 \mathrm{~b}$, which is then transformed into the phase-velocity spectrum depicted in Figure 3c. The blue dots corresponds to the measured curve and the red dots represent the actual phase-velocity curve (Lai and Glenn, 1998). The paucity of low-frequency information and long-offset traces prevented an accurate estimate of the phase velocities at low frequencies.

Discretizing the model into 5 homogeneous layers with the same thickness and each having an unknown S-wave velocity, the steepest descent algorithm after 50 iterations gives the phase-velocity curve in Figure $3 \mathrm{e}$ and the blue S-wave velocity profile in Figure $3 \mathrm{f}$.

\section{Inversion of Field Data}

A seismic land survey was carried out near the Red Sea coast in Saudi Arabia to give the recorded shot gather shown in Figure 4a. The geophone spacing is 5 meters, the source is a hammer on a metal plate, and the dominant frequency in the traces is about $40 \mathrm{~Hz}$. An FK transform is applied to the Figure 4a shot gather and the phase-velocity curve for the fundamental-mode is picked and displayed as the red dots in Figure 4c. Using a 5-layer velocity model, these phase velocities are inverted using the steepest descent formula in equation 2 to give the predicted blue points in Figure 4c. The P-wave velocity values were extracted from a $\mathrm{P}$-wave velocity tomogram, and a constant density model is assumed. Figure $4 d$ shows the initial velocity model depicted by the green curve and the inverted Swave velocity profile shown in Figure $4 d$ provides a reasonable range of $\mathrm{S}$ velocities for these types of sediments.

Extension to a 2D Medium

The skeletonized procedure for inverting the fundamental-mode 

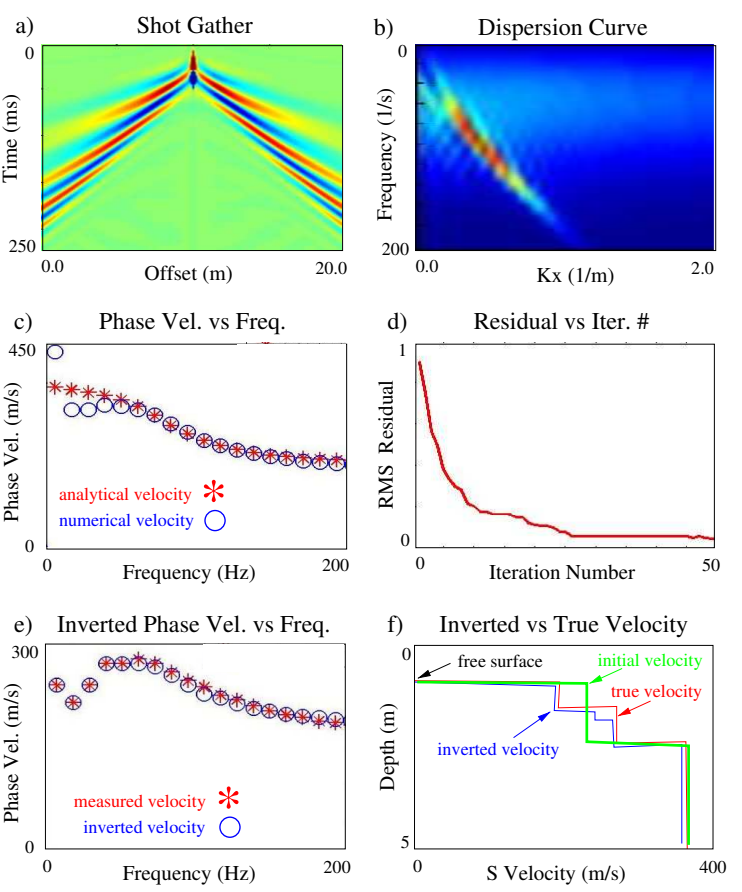

Figure 3: Surface-wave inversion results for synthetic data. a) CSG for the vertical particle-velocity traces $u(g, t)$, b) its Fourier transform $U(k, \omega)$, c) the analytical (red) and numerically estimated (blue) phase-velocity $C(\omega)$ values for the fundamental-mode in a 2-layer medium, d) normalized residual vs iteration number for inverting phase velocities computed for the 3-layer model in f), e) inverted (blue) and measured (red) phase velocity points for the 3-layer model in f), and f) inverted S-wave velocity model denoted by blue lines. The true model denoted by the red line in $\mathrm{f}$ ) consists of three layers below a free surface.
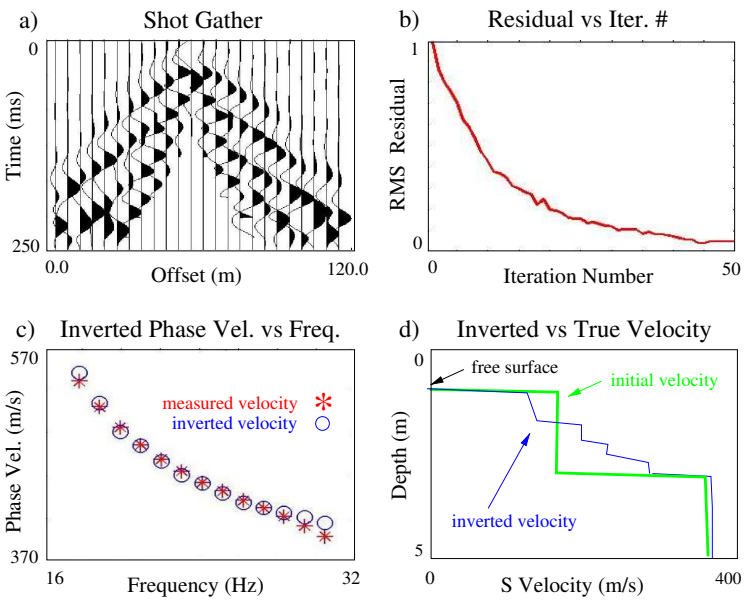

Figure 4: Surface wave inversion results for data recorded by a land survey near the Red Sea. a) CSG for the vertical particle velocity traces $u(g, t), \mathrm{b})$ residual vs iteration number, c) measured (red) and inverted (blue) phase-velocity values $C(\omega)$ for the fundamental mode, and d) inverted $\mathrm{S}$-wave velocity model (blue curve).

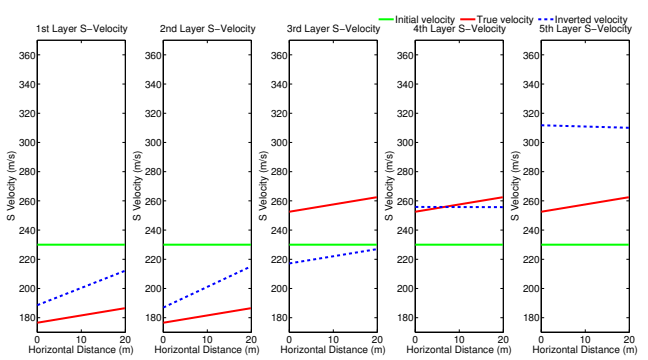

Figure 5: Surface-wave inversion results for inverting one shot gather (200 receivers/shot at 0.1 meter receiver spacing) with a dominant frequency of $80 \mathrm{~Hz}$. Inverted S-wave velocity model is denoted by blue lines, initial velocity model is denoted by green lines, and the true velocity model is denoted by red lines.
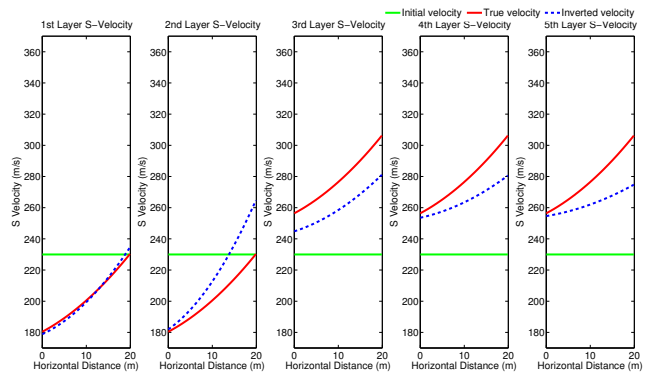

Figure 6: Same as Figure 5, except 3 shot gathers are simultaneously inverted. The shots are separated by 2.3 meters.

of surface waves can be extended to models with lateral Swave velocity variations by assuming a quadratic velocity variation $c(x)_{i}$ in the $i^{\text {th }}$ layer:

$$
c(x)_{i}=c_{i}+\gamma_{i} x+\zeta_{i} x^{2}
$$

where $c_{i}, \gamma_{i}$ and $\zeta_{i}$ are unknown constants that are to be inverted for by the steepest descent formula 2 . Instead of inverting for just one unknown in each layer, three unknowns are to be inverted which triples the computational cost. However, the total computational cost is quite affordable for velocity profiles with no more than several dozen layers. As an example, Figure 5 depicts the actual and predicted lateral velocity variations using the steepest descent method to invert for $\gamma_{i}$ in each layer for one shot gather. These preliminary results suggest that the surface wave data are somewhat insensitive to lateral velocity variations for just one shot gather. However, if three shot gathers are inverted with acceptable errors then the lateral velocity variations are reconstructed as shown in Figures 6 and 7.

\section{Sensitivity Matrix}

Finally, a sensitivity matrix can easily be computed to assess the sensitivity of the data to changes in the S-wave velocity model. The elements of the sensitivity matrix are the computed Fréchet derivatives $\frac{\partial C\left(\omega_{j}\right)_{i}}{\partial c_{j}}$ for different frequencies and layer numbers. As an example, Figure 8 plots these values for the 5-layer model in Figure 4d and shows that for the available frequency band, the dispersion curve is more sensitive to the 


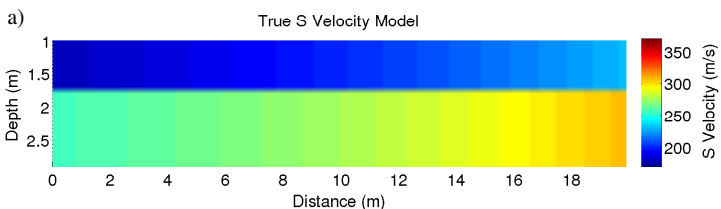

b)
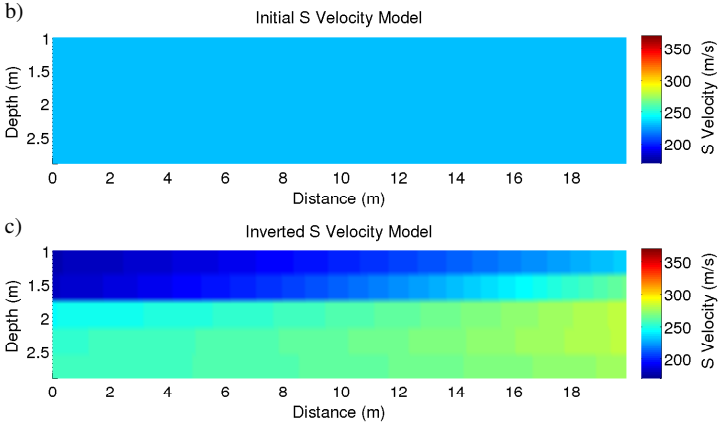

Figure 7: 2D inversion results. a) True S-wave velocity model, b) initial S-wave velocity model which is a constant velocity model and c) inverted S-wave velocity model.

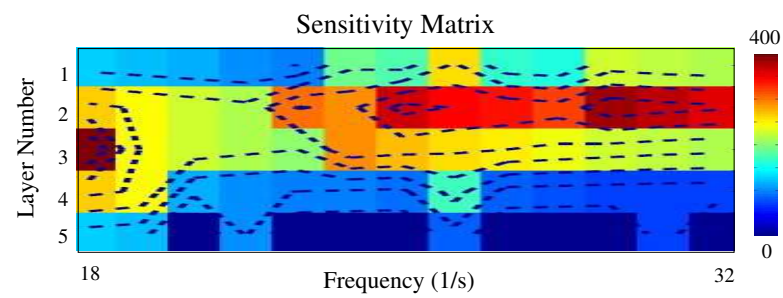

Figure 8: Sensitivity matrix of Fréchet derivative values for the 5-layer model in Figure 4d. As expected, higher frequencies in the data are mainly influenced by the S-velocities in specified layers.

velocity variations in the middle layers. Thus, these are the layers which should provide the least uncertainty in velocity reconstruction.

\section{CONCLUSION}

We presented a wave-equation method for inverting the dispersion curves associated with surface waves. The main benefits of this approach are that it mitigates cycle skipping problems associated FWI of surface waves, it is efficient for a limited number of layers, and is applicable to $2 \mathrm{D}$ and $3 \mathrm{D}$ velocity models. In our example, the dispersion curve for the fundamental mode is automatically picked and inverted by a steepest descent method in conjunction with finite-difference solutions to the wave equation. Higher-order modes can also be picked and inverted. Results for both synthetic data and field data verify the effectiveness of this method and reveal some of its limitations. Future research should test this method on more complex models; we also need to determine the optimal model parameterization that avoids the generation of an unde- termined system of equations. One of the implicit assumptions is that the S-velocity model is simple enough to produce a fundamental Rayleigh curve that is easily pickable. This assumption will be violated in more complex models and make it difficult to implement the skeletonized inversion method. We have also developed the analytic formulas (not shown) for implementing skeletonized surface wave inversion with the adjoint state method (Schuster, 2015). Implementing these formulas is a topic of future research. 


\section{EDITED REFERENCES}

Note: This reference list is a copyedited version of the reference list submitted by the author. Reference lists for the 2015 SEG Technical Program Expanded Abstracts have been copyedited so that references provided with the online metadata for each paper will achieve a high degree of linking to cited sources that appear on the Web.

\section{REFERENCES}

Aki, K., and P. Richards, 1980, Quantitative seismology: W. H. Freeman and Company.

Baumstein, A., W. Ross, and S. Lee, 2011, Simultaneous source elastic inversion of surface waves: 73rd Conference \& Exhibition, EAGE, Extended Abstracts, C040.

Dong, Z., C. Xiaofei, and M. Xiaogui, 2014, Rayleigh wave analysis and inversion for near surface shear wave velocity model building: Presented at the Beijing International Geophysical Conference and Exposition, 1217-1220.

Gabriels, P., R. Snieder, and G. Nolet, 1987, In situ measurements of shear-wave velocity in sediments with higher-mode Rayleigh waves: Geophysical Prospecting, 35, no. 2, 187196, http://dx.doi.org/10.1111/j.1365-2478.1987.tb00812.x.

Lai, C. G., and J. R. Glenn, 1998, Simultaneous inversion of Rayleigh phase velocity and attenuation for near-surface site characterization: Ph.D. dissertation, Georgia Institute of Technology.

Luo, Y., and G. T. Schuster, 1991a, Wave equation inversion of skeletalized geophysical data: Geophysical Journal International, 105, no. 2, 289-294, http://dx.doi.org/10.1111/j.1365246X.1991.tb06713.x.

Luo, Y., and G. T. Schuster, 1991b, Wave-equation traveltime inversion: Geophysics, 56, 645653, http://dx.doi.org/10.1190/1.1443081.

McMechan, G. A., and M. J. Yedlin, 1981, Analysis of dispersive waves by wave field transformation: Geophysics, 46, 869-874, http://dx.doi.org/10.1190/1.1441225.

Park, C. B., R. D. Miller, and J. Xia, 1998, Imaging dispersion curves of surface waves on multi-channel record: 68th Annual International Meeting, SEG, Expanded Abstracts, 1377-1380.

Pérez Solano, C. A., D. Donno, and H. Chauris, 2014, Alternative waveform inversion for surface wave analysis in 2-D media: Geophysical Journal International, 198, no. 3, 13591372, http://dx.doi.org/10.1093/gji/ggu211.

Virieux, J., and S. Operto, 2009, An overview of full-waveform inversion in exploration: Geophysics, 74, no. 6, WCA4-WCA17, http://dx.doi.org/10.1190/1.3238367.

Xia, J., R. D. Miller, and C. B. Park, 1999, Estimation of near-surface shear-wave velocity by inversion of Rayleigh waves: Geophysics, 64, 691-700, http://dx.doi.org/10.1190/1.1444578. 